\title{
Globally rigid augmentation of minimally rigid graphs in $\mathbb{R}^{2}$
}

\author{
Csaba Király ${ }^{1,2[0000-0001-8081-9056]}$ and András \\ Mihálykó $2[0000-0002-0624-655 X]^{2}$ \\ 1 MTA-ELTE Egerváry Research Group, Budapest, Hungary \\ 2 Department of Operations Research, ELTE Eötvös Loránd University, \\ Budapest, Hungary \\ \{cskiraly, mihalyko\}@cs.elte.hu
}

\begin{abstract}
The two main concepts of Rigidity Theory are rigidity, where the framework has no continuous deformation, and global rigidity, where the given distance set determines the locations of the points up to isometry. We consider the following augmentation problem. Given a minimally rigid graph $G=(V, E)$ in $\mathbb{R}^{2}$, find a minimum cardinality edge set $F$ such that the graph $G^{\prime}=(V, E+F)$ is globally rigid in $\mathbb{R}^{2}$. We provide a min-max theorem and an $O\left(|V|^{2}\right)$ time algorithm for this problem.
\end{abstract}

Keywords: Global Rigidity · Augmentation · Rigidity · Combinatorial Algorithm

\section{Introduction}

Let us consider the following motivating question: Given some sensors in the plane and the distances between some pairs of them, at least how many of them need to be localized so that we could reconstruct the exact sensor-locations? This is the so-called global rigidity pinning (or anchoring) problem. Sometimes measuring the exact locations is too expensive or even impossible. Instead, one may ask at least how many new distances need to be measured so that the distances uniquely determine the positions of the sensors (up to isometry). This problem is called the global rigidity augmentation problem. The concept of global rigidity, which appears in the previous network localization problems, plays an important role in rigidity theory $[3,5,12]$.

Let us consider the aforementioned problems by the means of Rigidity Theory. A $d$-dimensional framework is a pair $(G, p)$, where $G=(V, E)$ is a graph and $p: V \rightarrow \mathbb{R}^{d}$ is a map of the vertices to the $d$-dimensional space. We call $p$ a realization of $G$ in $\mathbb{R}^{d}$. Two frameworks $(G, p)$ and $(G, q)$ are equivalent if $\|p(u)-p(v)\|=\|q(u)-q(v)\|$ for every $u v \in E$. $(G, p)$ and $(G, q)$ are congruent if $\|p(u)-p(v)\|=\|q(u)-q(v)\|$ holds for every vertex pair $u, v \in V$, or in other words, when $(G, q)$ can be obtained from $(G, p)$ by an isometry of $\mathbb{R}^{d}$. We say that the framework $(G, p)$ is globally rigid if each framework $(G, q)$ which is equivalent to $(G, p)$ is also congruent to $(G, p)$, that is, the length of the edges in $(G, p)$ uniquely determines the realization up to isometry of $\mathbb{R}^{d}$. (For 
example, Figure $1(\mathrm{c})$ is a globally rigid framework in $\mathbb{R}^{2}$.) A framework $(G, p)$ is called rigid if there exists an $\varepsilon>0$ such that each framework $(G, q)$, which is equivalent to $(G, p)$ and for which $\|p(v)-q(v)\|<\varepsilon$ holds for each $v \in V$, is also congruent to $(G, p)$, that is, if every edge-length preserving continuous motion of the framework results in a framework which is congruent to $(G, p)$. (See Figure 1(a) for an example of a non-rigid and Figure 1(b) for a rigid framework in $\mathbb{R}^{2}$.)

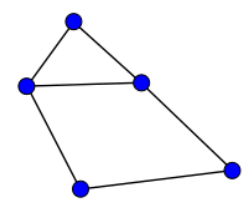

(a)

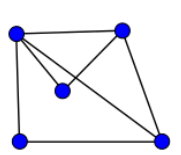

(b)

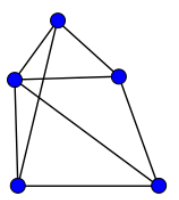

(c)

Fig. 1: Frameworks of various rigidity in $\mathbb{R}^{2}$. (a) A non-rigid framework. (b) A rigid framework which is not globally rigid. (c) A globally rigid framework.

Deciding whether a given framework is rigid (globally rigid, respectively) in $\mathbb{R}^{d}$ is NP-hard for $d \geq 2(d \geq 1$, respectively) [1,21]. The analysis gets more tractable if we consider generic frameworks where the set of coordinates of the points is algebraically independent over the rationals. In this case, the rigidity and the global rigidity of the framework depends only on the underlying graph $G$ $[5,8,23]$. (We note that reconstructing the position of the points is a challenging task, even if they are uniquely determined by the framework, see $[2,16,22]$. In this paper we do not address this problem.)

A graph $G$ is called rigid (or globally rigid) in $\mathbb{R}^{d}$ if each (or equivalently some) of its generic realizations as a framework is rigid (or globally rigid, respectively). The combinatorial characterization of rigid and globally rigid graphs is known for $d=1,2[11,20]$ while it is a major open problem of rigidity theory for $d \geq 3$. We shall use these combinatorial characterizations in our work.

For generic frameworks, the global rigidity augmentation problem can be modelled as follows:

Problem 1 Given a graph $G=(V, E)$, find an edge set $F$ of minimum cardinality on the same vertex set, such that $G+F=(V, E \cup F)$ is globally rigid in $\mathbb{R}^{2}$.

The complexity of Problem 1 is open. There are some partial results in connection with it, for example, Fekete and Jordán [6] gave a constant factor approximation for the global rigidity pinning problem in $\mathbb{R}^{2}$ for generic frameworks, however, the complexity of that problem is also open. In Section 5 we show how the result of [6] can be applied to give a constant factor approximation for Problem 1.

In this paper we shall solve Problem 1 optimally for a special case. A graph $G=(V, E)$ is called minimally rigid, if $G$ is rigid but $G-e$ is not rigid for any 
$e \in E$. We show that, if $G$ is minimally rigid in Problem 1 , then we can give a min-max theorem and also an $O\left(|V|^{2}\right)$ time algorithm that solves the problem optimally. Moreover, it follows from this result that the globally rigid pinning problem also can be solved optimally for minimally rigid graphs (see Section 5). The most of the proofs are left for the full version of this extended abstract [17].

\section{Preliminaries and Definitions}

\subsection{Rigidity in $\mathbb{R}^{2}$}

In this subsection we collect the basic definitions and results from rigidity theory that we shall use. There are several equivalent approaches to graph rigidity, for our purpose, a combinatorial one is the most practical. For a detailed introduction to rigidity theory including the equivalence of our approach, the reader is referred to [14].

A graph $G=(V, E)$ is called sparse if $i(X) \leq 2|X|-3$ for all $X \subseteq$ $V$ with $|X| \geq 2$, where $\boldsymbol{i}(\boldsymbol{X})$ denotes the number of edges induced by $X$. A graph $G=(V, E)$ is called tight (or sometimes Laman) if it is sparse and $|E|=2|V|-3$. This definition can be used for the characterization of the rigid graphs in $\mathbb{R}^{2}$ by the fundamental results of Pollaczek-Geiringer and Laman.

Theorem 1 ([20],[19]). A graph $G$ is minimally rigid in $\mathbb{R}^{2}$ if and only if $G$ is tight. Thus, a graph $G$ is rigid in $\mathbb{R}^{2}$ if it contains a spanning tight subgraph.

As we work in $\mathbb{R}^{2}$ we omit this indication from the rest of this paper. A graph $G=(V, E)$ is called $\boldsymbol{k}$-connected if $|V|>k$ and $G-X$ is connected for any vertex set $X \subset V$ of cardinality at most $k-1$. Connectivity has several connections to rigidity. An often used folklore result is the following (see [14]).

Lemma 1. If $G=(V, E)$ is a tight graph for which $|V| \geq 3$, then $G$ is 2connected.

The most important result related to our problem is the following characterization of global rigidity in $\mathbb{R}^{2}$ due to Jackson and Jordán. An edge $e$ of a rigid graph $G$ is called redundant if $G-e$ is rigid. A graph is redundantly rigid if all of its edges are redundant.

Theorem 2 ([11]). A graph $G=(V, E)$ with $|V|>3$ is globally rigid in $\mathbb{R}^{2}$ if and only if it is redundantly rigid and 3-connected.

Based on the above results, the problem we shall solve in this paper is equivalent to the following.

Problem 2 Given a tight graph $G=(V, E)$, find a graph $H=(V, F)$ with a minimum cardinality edge set $F$, such that $G \cup H$ is redundantly rigid and 3-connected.

If $G$ has at most 3 vertices then $G$ is tight if and only if it is globally rigid [11], hence the solution of Problem 2 is obvious. Thus we may suppose in what follows that $G$ contains at least 4 vertices. 


\subsection{The redundant rigidity augmentation problem and co-tight sets}

Let us first investigate the problem of augmenting a tight graph $G=(V, E)$ to a redundantly rigid graph by a minimum number of edges. This problem was considered and solved before by García and Tejel [7]. A generalization of this augmentation problem to $(k, \ell)$-tight graphs appears in a work by the authors of this paper [18]. We use some ideas from both of these works.

Tight graphs have some well known properties. By definition, any subgraph of a sparse graph is also sparse and any tight subgraph of a sparse graph is an induced subgraph. With standard submodular techniques one can prove the well-known fact that the intersection and the union of two tight subgraphs of a sparse graph is also tight if they have at least two common vertices (see [14]). Given two vertices $u, v \in V$ of a tight graph $G=(V, E)$, this fact implies that the intersection of all the tight subgraphs of $G$ which contain both of $u$ and $v$ is also tight, and hence it is the unique minimal tight subgraph of $G$ containing both of $u$ and $v$. Let us denote this unique minimal tight subgraph of $G$ containing both of $u$ and $v$ by $\boldsymbol{T}(\boldsymbol{u} \boldsymbol{v})$ (or simply by $T(e)$ when $e$ is an edge between $u$ and $v)$. It is easy to see that the edge set of $T(e)$ is exactly the set of those edges of $G$ which become redundant if we add the edge $e$ to $G$ (see [7]). Similarly, if we add the edges $e_{1}, \ldots, e_{k}$ to $G$, (the edges of) some subgraph of $G$ will become redundant, which we denote by $\boldsymbol{R}\left(e_{1}, \ldots, e_{k}\right)$. For the sake of convenience, we will not distinguish a graph from its edge set, that is, we denote the edge set of $T(e)$ and $R\left(e_{1}, \ldots, e_{k}\right)$ by $T(e)$ and $R\left(e_{1}, \ldots, e_{k}\right)$, respectively. The following statement generalizes the fact that $R\left(e_{1}\right)=T\left(e_{1}\right)$.

Lemma 2 ([7, Lemma 4]). Let $G=(V, E)$ be a tight graph. Then $R\left(e_{1}, \ldots, e_{k}\right)=$ $T\left(e_{1}\right) \cup \cdots \cup T\left(e_{k}\right)$ for arbitrary edges $e_{1}, \ldots, e_{k}$.

Lemma 2 is the base of our method hence we will use it throughout the paper without explicitly referring to it.

Given a tight graph $G=(V, E)$, a non-empty set $C \subsetneq V$ is called co-tight if $V-C$ induces a tight subgraph. This is equivalent to the following: $C$ is co-tight in $G$ if $0<|C| \leq|V|-2$ and $2|C|=i(C)+d(C, V-C)$, where $\boldsymbol{d}(\boldsymbol{X}, \boldsymbol{Y})$ denotes the number of edges between two disjoint sets $X, Y \subsetneq V$. For the sake of brevity, let us abbreviate the name of minimal co-tight sets by MCT sets. See Figure 2 for an example. Observe that every tight graph $G$ on at least 4 vertices contains at least two co-tight sets that do not contain each other, as any edge forms a tight subgraph of $G$.

Let $C$ be a co-tight set of a tight graph $G$. If $\{u, v\} \cap C=\emptyset$, then $V(T(u v)) \cap$ $C=\emptyset$ by the definition of $T(u v)$. Thus the next lemma follows easily by Lemma 2.

Lemma 3 ([18, Observation 5.3]). The vertex set of any edge set that augments a tight graph $G$ to a redundantly rigid graph must intersect every co-tight set.

Let $\mathcal{C}^{*}$ denote the family of all MCT sets of $G$. We shall use the following key result on MCT sets (which are called minimal co-rigid sets in [14]). 


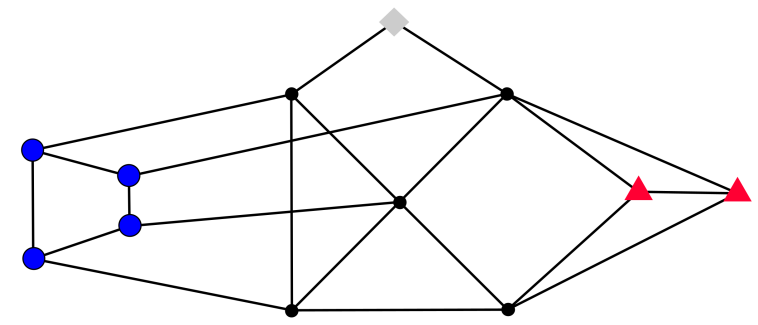

Fig. 2: A tight graph with two MCT sets, the set formed by the big (blue) circles and the set formed by the (gray) square. Adding an edge between any (blue) circle vertex and the (gray) square vertex augments $G$ to a redundantly rigid graph, which is not globally rigid, as it is not 3-connected. Adding an edge between a (red) triangle vertex and the (gray) square vertex augments $G$ to a 3 -connected but not redundantly rigid graph.

Lemma 4 ([14, Theorem 3.9.13]). Let $G$ be a tight graph. Then the members of $\mathcal{C}^{*}$ are pairwise disjoint or there are two vertices $v, w \in V$ such that $\{v, w\} \cap$ $C \neq \emptyset$ for all $C \in \mathcal{C}^{*}$.

If there are at least two intersecting MCT sets, then it is easy to deduce from Lemma 4 that the edge $e=v w$ (for the pair $v, w \in V$ provided by the lemma) is an optimal solution of the redundant augmentation problem, that is, $R(e)=$ $T(e)=G$. In the general case, the following theorem determines the cardinality of the optimal augmentation.

Theorem 3 ([18, Theorem 1.1]). Let $G$ be a tight graph on at least 4 vertices. Then $\min \{|F|: F$ is an edge set on $V$ for which $G+F$ is a redundantly rigid graph $\}=\max \left\{\left\lceil\frac{|\mathcal{C}|}{2}\right\rceil: \mathcal{C}\right.$ is a family of disjoint co-tight sets in $\left.G\right\}$.

\subsection{The 3-connectivity augmentation problem}

By Lemma 1, every tight graph is 2-connected and thus we need to augment a 2-connected graph to a 3-connected graph. There exists several methods to deal with this particular problem, even linear time algorithms [10]. However, we also need to augment $G$ to a redundantly rigid graph hence we stick to a simpler approach following the ideas of [13].

Let us call $u, v \in V$ a cut-pair of $G$, if $G-\{u, v\}$ is not connected. If $u, v$ is a cut-pair in $G$, then let $\boldsymbol{b}_{(u, v)}(\boldsymbol{G})$ denote the number of components of $G-\{u, v\}$. Let $\boldsymbol{b}(\boldsymbol{G})$ denote the maximum value of $b_{(u, v)}(G)$ over all cut-pairs $u, v$ of $G$. If there are no cut-pairs in $G$, let $b(G):=1$. Let $\boldsymbol{N}(\boldsymbol{X})$ denote the neighbor set of $X \subseteq V$, that is, $N(X):=\{v \in V-X$ : there exists an edge $u v$ such that $u \in X\}$. A set $P \subset V$ is called a 3-fragment if $|N(P)|=2$ and $P \cup N(P) \neq V$. The maximum number of pairwise disjoint 3-fragments is denoted by $\boldsymbol{t}(\boldsymbol{G})$. 
To augment a 2-connected graph $G$ to a 3 -connected graph, we need to increase the number of neighbors of each 3 -fragment of $G$, and hence the vertex set of any edge set that augments $G$ to a 3-connected graph must intersect all 3 -ends. Moreover, any edge set $F$ that augments $G$ to a 3-connected graph needs to span a connected graph on the components of $G-\{u, v\}$ for every cut-pair $u, v$. Thus $|F| \geq b(G)-1$. These imply the following well-known statement.

Lemma 5. Given a 2-connected graph $G$, the minimum number of edges that augments $G$ to a 3-connected graph is at least $\max \left\{b(G)-1,\left\lceil\frac{t(G)}{2}\right\rceil\right\}$.

In fact, any 2-connected graph can be augmented to a 3-connected graph by a set of $\max \left\{b(G)-1,\left[\frac{t(G)}{2}\right]\right\}$ edges (see $[10,13]$ ).

Let us call an inclusion-wise minimal 3-fragment a 3-end. As every 3-fragment contains at least one 3-end, $t(G)$ is equal to the number of pairwise disjoint 3ends. In a rigid graph, this latter value is equal to the number of 3 -ends since their disjointness follows by the following result of Jackson and Jordán [11].

Lemma 6 ([11]). Let $G$ be a rigid graph in $\mathbb{R}^{2}$. Then, for any two disjoint cutpairs $v_{1}, v_{2}$ and $u_{1}, u_{2}$ of $G, u_{1}$ and $u_{2}$ are in the same component of $G-\left\{v_{1}, v_{2}\right\}$.

\section{Min-max theorem}

In this section we shall merge the results on the redundant rigidity and 3connectivity augmentation problems to a new min-max theorem for the global rigidity augmentation problem by mixing the statements of Theorem 3 and Lemma 5, as follows.

Theorem 4. Let $G=(V, E)$ be a tight graph on at least 4 vertices. Then $\min \{|F|: F$ is an edge set on $V$ for which $G+F$ is globally rigid $\}=\max \{b(G)-$ $1, \max \left\{\left\lceil\frac{|\mathcal{A}|}{2}\right\rceil: \mathcal{A}\right.$ is a family of disjoint co-tight sets and 3 -fragments $\left.\}\right\}$.

Proof (Sketch). Recall that a graph on at least 4 vertices is globally rigid if and only if it is 3 -connected and redundantly rigid by Theorem 2 . The $\min \geq \max$ implication in Theorem 4 is obvious since the set of endvertices of the optimal augmenting edge set must intersect all co-tight sets and 3-fragments by Lemmas 3 and 5. Notice that, if $G$ is 3 -connected, then Theorem 4 follows directly by Theorem 3. Hence from now on, we may assume that $G$ is not 3 -connected. In this case we shall extend the proof of Theorem 3 given in [18] with the ideas of the 3-connectivity augmentation method given by Jordán [13]. Hence to prove the min $\leq \max$ part, let us consider the family of all MCT sets and 3-ends of a tight graph $G$. Let us call the inclusion-wise minimal elements of this family the atoms of $G$. (In Figure 2 these are the three sets formed by the highlighted vertices: the big (blue) circles form an MCT set, the (gray) square vertex form an MCT set which is also a 3-end, and the (red) triangle vertices form a 3-end.) Let 
us denote the family of atoms by $\mathcal{A}^{*}$. We shall show that the atoms are pairwise disjoint and there exists a set of $\max \left\{b(G)-1,\left\lceil\frac{\left|\mathcal{A}^{*}\right|}{2}\right\rceil\right\}$ edges that augments $G$ to a globally rigid graph. Hence we first need the following counterpart of Lemma 4 for atoms.

Lemma 7. Let $G=(V, E)$ be a tight graph which is not 3-connected. Then the atoms of $G$ are pairwise disjoint.

Note that if $G$ is 3 -connected, Lemma 7 does not always hold (see Lemma 4). As we have seen before in Section 2.3, the 3-ends of $G$ are pairwise disjoint and Lemma 4 implies that two MCT sets can only intersect each other in special circumstances. Beside these facts, the proof of Lemma 7 uses the following intermediate result. The proofs of both lemmas can be found in the full version [18] of this extended abstract.

Lemma 8. Suppose that $G=(V, E)$ is a tight graph. Let a $\in A$ be a vertex from an atom $A \in \mathcal{A}^{*}$ of $G$. Then there is no $v \in V$ such that a, $v$ forms a cut-pair.

Now, we turn to prove that there exists a set of $\max \left\{b(G)-1,\left\lceil\frac{\left|\mathcal{A}^{*}\right|}{2}\right\rceil\right\}$ edges that augments $G$ to a globally rigid graph. A set $X$ is called a transversal of a family $\mathcal{S}$ if $|X \cap S|=1$ for each $S \in \mathcal{S}$ and $|X|=|\mathcal{S}|$. As the members of $\mathcal{A}^{*}$ are pairwise disjoint if $G$ is not 3 -connected by Lemma 7 , choosing one arbitrary vertex from every member of $\mathcal{A}^{*}$ leads to a transversal of $\mathcal{A}^{*}$.

Let $P$ be a transversal of $\mathcal{A}^{*}$. Observe that $P$ is a minimum cardinality vertex set that intersects all MCT sets and 3-ends, and consequently all co-tight sets and 3-fragments. Hence $|\mathcal{A}| \leq|P|$ holds for an arbitrary family $\mathcal{A}$ of disjoint cotight sets and 3 -fragments. We shall show now that a connected graph on $P$ of $\mathcal{A}^{*}$ augments $G$ to a globally rigid graph, that is, 3-connected and redundantly rigid. Later, we will reduce the number of edges needed for this augmentation to the optimum value. First it is easy to observe that any connected graph on $P$ augments $G$ to a 3 -connected graph since $P$ covers all 3 -ends (by the definition of the atoms and Lemma 7 ) and contains no vertex from any cut-pair by Lemma 8.

Lemma 9. Suppose that $G$ is a tight graph which is not 3-connected. Let $P$ be a transversal of $\mathcal{A}^{*}$. Then, for any connected graph $H=(P, F)$ on $P, G \cup H$ is 3-connected.

To show that the above augmentation gives a redundantly rigid graph, one can extend the ideas of the proof of Theorem 3 from [18] for atoms by using Lemma 7 instead of Lemma 4. (Again, see [17] for the full proofs.) Recall that $R(F)$ denotes the set of redundant edges of $G$ in $G+F$.

Lemma 10 (Extension of [18, Lemma 5.8]). Suppose that $G$ is a tight graph which is not 3-connected. Let $P$ be a transversal of $\mathcal{A}^{*}$ and let $F$ be the edge set of a connected graph on $P^{\prime} \subseteq P$. Then $R(F)$ is the minimal tight subgraph containing all elements of $P^{\prime}$. In particular, if $F$ is the edge set of a star $K_{1,|P|-1}$ on the vertex set $P$, then $G+F$ is redundantly rigid. 
Observation 1 Lemmas 9 and 10 imply that $G+F$ is globally rigid if $F$ is an edge set of an arbitrary connected graph (in particular, a tree) on a transversal $P$ of $\mathcal{A}^{*}$.

The idea of Observation 1 can be found in [15], where the authors got to this fact from a different approach, with the so-called extreme vertices. The connection between these two approaches is presented in [18, Lemma 5.10].

By the min $\geq$ max part of Theorem $4,\left[\frac{\left|\mathcal{A}^{*}\right|}{2}\right]$ edges are always needed to augment $G$ to a globally rigid graph. However, if $\left|\mathcal{A}^{*}\right| \leq 3$ then it is indeed enough to do so by Observation 1 . On the other hand, if $\left|\mathcal{A}^{*}\right|>3$, then we need to reduce the number of edges used by the augmentation provided by Observation 1 . To this end, we shall use the following straightforward adaptation of [18, Lemma 5.9] (see [17] for the proof).

Lemma 11. Let $G=(V, E)$ be a tight graph which is not 3-connected and let $P$ be a transversal of $\mathcal{A}^{*}$. Suppose that $x_{1}, x_{2}, x_{3}, y \in P$ are distinct vertices. Let $T^{*}=T\left(x_{1} y\right) \cup T\left(x_{2} y\right) \cup T\left(x_{3} y\right)$. Then $T^{*}=T\left(x_{1} y\right) \cup T\left(x_{2} x_{3}\right)$ or $T^{*}=$ $T\left(x_{2} y\right) \cup T\left(x_{1} x_{3}\right)$ holds.

Observe that the operation in Lemma 11 allows us to reduce the cardinality of the edge set used for the augmentation by maintaining the property that it augments $G$ to a redundantly rigid graph. However, we also need to maintain the 3-connectivity of the augmentation to complete the proof of Theorem 4 .

To reduce the number of edges needed for the augmentation in such a way that the global rigidity of the augmented graph is maintained, we do the following procedure. Initially, let $F:=\emptyset$ and $N:=P$. During the procedure, the set $N \subseteq P$ stands for "not fixed" vertices while vertices in $P-N$ are the "fixed" vertices. We can fix an edge $f_{1} f_{2}$ by removing $f_{1}$ and $f_{2}$ from $N$ and adding $f_{1} f_{2}$ to $F$. In each step of the procedure we carefully choose two vertices from $N$ and fix the edges between them (decreasing the number of vertices in $N$ by two and increasing the number of edges in $F$ ). Hence the edge set $F$ always covers the vertices of $P-N$. We shall keep the following properties during the whole procedure:

1. For an arbitrary star $\boldsymbol{S}_{N}$ on the vertex set $N, G+F+S_{N}$ is a redundantly rigid graph.

2. In every 3-end of $G+F$, there is at least one vertex from $N$

3. $\max \left\{b(G+F)-1,\left\lceil\frac{|N|}{2}\right\rceil\right\}+|F|=\max \left\{b(G)-1,\left\lceil\frac{|P|}{2}\right\rceil\right\}$.

Notice that Properties 1-3 hold for $N=P$ and $F=\emptyset$ by Lemmas 9 and 10 .

Remark 1 Properties 1 and 2 ensure that $G+F+S_{N}$ is redundantly rigid and 3-connected, and thus globally rigid by Theorem 2. Property 3 ensures the optimality.

Remark 2 If $|N| \geq 4$, then from any two edges chosen on $x_{1}, x_{2}, x_{3} \in N$ fixing one of them maintains Property 1 by Lemma 11. 
By Remark 2 we always aim to find at least two possibilities to fix such that Property 2 holds. Also, if it can be done so that $\max \left\{b(G+F)-1,\left\lceil\frac{|N|}{2}\right\rceil\right\}$ decreases by one, then we can maintain Properties 1-3. Roughly, we distinguish 4 different possibilities in each of which we find 3 vertices from $N$ such that we can apply Remark 2 and hence we can fix one edge while maintaining Properties $1-3$.

Lemma 12. Let $G$ be a tight graph which is not 3-connected such that $\left|\mathcal{A}^{*}\right| \geq 4$. Let $P$ be a transversal on $\mathcal{A}^{*}$. Let $N \subseteq P$ be a vertex set and $F$ be an edge set on $P$ such that they satisfy Properties 1-3. If $|N| \geq \max \{4, b(G+F)+1\}$, then we can choose $f_{1}, f_{2} \in N$, such that for $N-\left\{f_{1}, f_{2}\right\}$ and $F+\left\{f_{1} f_{2}\right\}$ (that is, for fixing $f_{1} f_{2}$ ) Properties 1-3 also hold.

Proof. We use the following method for the proof. This is the core of our algorithm which we will describe in Section 4.

1 If $b(G+F)-1 \geq\left\lceil\frac{|N|}{2}\right\rceil$, then

2 If there is only one cut-pair $(u, v)$ such that $b_{(u, v)}(G+F)=b(G+F)$, then

Choose $x_{1}, x_{2}$ from a component of $G+F-\{u, v\}$ that contains at least two vertices from $N$. Let $x_{3} \in N$ be a vertex from a component of $G+F-\{u, v\}$ that does not contain $x_{1}$ and $x_{2}$.

3 else

Let $\left(u_{1}, v_{1}\right)$ and $\left(u_{2}, v_{2}\right)$ be two cut-pairs for which $b_{\left(u_{1}, v_{1}\right)}(G+F)=$ $b(G+F)=b_{\left(u_{2}, v_{2}\right)}(G+F)$. Choose $x_{1}, x_{2} \in N$ from two different components of $G+F-\left\{u_{1}, v_{1}\right\}$ that do not contain $\left\{u_{2}, v_{2}\right\}$. Choose $x_{3} \in N$ from a component of $G+F-\left\{u_{2}, v_{2}\right\}$ that does not contain $\left\{u_{1}, v_{1}\right\}$.

4 else

5 If there is a cut-pair $\{u, v\}$ such that for one component of $G+F-\{u, v\}$, say $K,|N \cap K| \geq 2$ and $|(V-K) \cap N| \geq 2$, then

Choose $x_{1}, x_{2}$ from $N \cap K$ and choose $x_{3} \in N$ from $(V-K) \cap N$.

6 else (Notice that if $b(G+F)=1$, then this is the only possible case.) Choose $x_{1}, x_{2}, x_{3} \in N$ arbitrarily.

7 If $G+F+S\left(N-\left\{x_{1}, x_{3}\right\}\right)+x_{1} x_{3}$ is redundantly rigid, then

else $f_{1}:=x_{1}, f_{2}:=x_{3}$.

$$
f_{1}:=x_{2}, f_{2}:=x_{3}
$$

First we prove that the above method is consistent, that is, we can execute each of its steps. As $|N| \geq b(G+F)+1$ and $P$ contains no vertex from a cut-pair of $G$ by Lemma $8,|N|>b_{(u, v)}(G+F)$ for an arbitrary cut-pair $\{u, v\}$. Hence, there exists a component of $G+F-\{u, v\}$ that contains at least two vertices from $N$. This shows that we can choose vertices in STEPs $\mathbf{2}$ and $\mathbf{5}$ consistently. Meanwhile, in STEP 3 there are at least two components of $G+F-\left\{u_{1}, v_{1}\right\}$ that do not contain $\left\{u_{2}, v_{2}\right\}$ since $|N| \geq 4$ and thus $b_{\left(u_{1}, v_{1}\right)}(G+F) \geq 3$.

Now let us turn to show that the choice of $f_{1}$ and $f_{2}$ maintains Property 2. 
Claim. Suppose that there is a cut-pair $\{u, v\}$ such that for one component of $G+F-\{u, v\}$, say $K, x_{1}, x_{2} \in N \cap K$ and $x_{3}, y \in(V-K) \cap N$. Then fixing either $x_{1} x_{3}$ or $x_{2} x_{3}$ maintains Property 2 .

Proof. Notice that the role of $x_{1}$ and $x_{2}$ is symmetric thus we might suppose that we fixed the edge $x_{1} x_{3}$. Suppose that we form a new 3 -end $L$ in $G+F$ with it. Then necessarily $x_{1}, x_{3} \in L$. If $x_{2} \in L$ or $y \in L$, then Property 2 holds automatically. On the other hand, if none of them is in $L$, then there is a cut-pair in $K \cup\{u\}$ or in $K \cup\{v\}$ which separates $x_{1}$ from $x_{2}$. There is another cut-pair in $V-K$ (other than $\{u, v\}$, say $\left\{u^{\prime}, v^{\prime}\right\}$ ) which separates $x_{3}$ from $y$. Both remain cut-pairs after fixing the edge $x_{1} x_{3}$. However, this contradicts the assumption that $L$ is 3-end in $G+F$, as $|N(L)|=2$ must hold for a 3-end.

Notice that the conditions of the above Claim hold in Steps 2, 3 and $\mathbf{5}$ thus with our choice of $x_{1}, x_{2}$, and $x_{3}$ Property 2 is maintained. If $G+F$ is already 3-connected, then Property 2 is obvious. Otherwise, in SteP 6, every cut-pair cuts $G+F$ into two component, one of which contains exactly one element from $N$ by the condition of STEP $\mathbf{5}$. For the sake of a contradiction, assume that $G+F+f_{1} f_{2}$ contains a 3 -end $L$ which contains no element of $N-\left\{f_{1}, f_{2}\right\}$. Let $N(L)=\{u, v\}$. Then $N \cap L=\left\{f_{1} . f_{2}\right\}, V-L-\{u, v\} \neq \emptyset$, and $u, v$ is a cut pair of $G+F$. By our above condition, $(u, v)$ cuts $G+F$ into two component one of which contains exactly one element from $N$. Hence exactly $L$ and $V-L-\{u, v\}$ are these two components. Moreover, as $|L \cap N|=2$, this implies $|N \cap(V-L-\{u, v\})|=1$, contradicting $|N| \geq 4$.

Now we show that our method maintains Property 3. Fixing any edge decreases $\left[\frac{|N|}{2}\right]$ by one while increases $F$ by one. By STEPS 5 and 6 it is enough to keep Property 3 true as in this case $\max \left\{b(G+F)-1,\left[\frac{|N|}{2}\right]\right\}>b(G+F)-1$. We need to show that if the condition in STEP 1 is true, then we also decrease $b(G+F)$. If $b(G+F)-1 \geq\left\lceil\frac{|N|}{2}\right\rceil$, then there can be at most two cut-pairs of $G+F$ satisfying $b_{(u, v)}(G+F)=b(G+F)$ by a simple calculation on the number of 3-ends (see [13]). If there is only one, the pair $(u, v)$ chosen in STEP 2, then we only need to decrease $b_{(u, v)}(G+F)$. Since $x_{1} x_{3}$ and $x_{2} x_{3}$ both connect two different components of $G+F-\{u, v\}, b_{(u, v)}(G+F)$ decreases by one after fixing any of them. If there are exactly two such cut-pairs, $\left(u_{1}, v_{1}\right)$ and $\left(u_{2}, v_{2}\right)$ chosen in STEP 3, then we need to decrease $b_{\left(u_{1}, v_{1}\right)}(G+F)$ and $b_{\left(u_{2}, v_{2}\right)}(G+F)$ simultaneously. Again our choice of $x_{1} x_{3}$ and $x_{2} x_{3}$ guarantees this.

Therefore, by Remark 2 applied to STEP 7, fixing $f_{1} f_{2}$ maintains Properties $1-3$. This completes the proof of Lemma 12.

We apply Lemma 12 recursively until $|N|<\max \{4, b(G+F)+1\}$. To complete the proof of Theorem 4 , we need to show the following.

Claim. If $|N| \leq \max \{3, b(G+F)\}$, then, for an arbitrary star $S_{N}$ on $N, G+F+$ $S_{N}$ forms a globally rigid graph for which $|F|+\left|S_{N}\right|=\max \left\{b(G)-1,\left\lceil\frac{|P|}{2}\right\rceil\right\}$. 
Proof. $G+F+S_{N}$ is globally rigid by Remark 1. By Property 3 it is enough to show that $\max \left\{b(G+F)-1,\left\lceil\frac{|N|}{2}\right\rceil\right\}=\left|S_{N}\right|=|N|-1$. If $|N|=b(G+F)$, then $\max \left\{b(G+F)-1,\left\lceil\frac{|N|}{2}\right\rceil\right\}=|N|-1$ as $\left\lceil\frac{|N|}{2}\right\rceil \leq|N|-1$. On the other hand, if $|N|<b(G+F)$, then $2 \leq|N| \leq 3$ thus $\left\lceil\frac{|N|}{2}\right\rceil=|N|-1$.

Recall that $\mathcal{A}^{*}$ consists of pairwise disjoint MCT sets and 3-ends of $G$ and hence the maximum in Theorem 4 is at least $\max \left\{b(G)-1,\left\lceil\frac{\left|\mathcal{A}^{*}\right|}{2}\right\rceil\right\}$. On the other hand, the above claim implies that $G$ can be augmented to a globally rigid graph by an addition of an edge set of cardinality $\max \left\{b(G)-1,\left\lceil\frac{|P|}{2}\right\rceil\right\}=$ $\max \left\{b(G)-1,\left\lceil\frac{\left|\mathcal{A}^{*}\right|}{2}\right\rceil\right\}$. This completes the proof of Theorem 4 .

\section{Algorithmic aspects}

It is easy to see that the proof of Theorem 4 provides an algorithm for Problem 2 when the input tight graph $G=(V, E)$ is not 3-connected. On the other hand, the algorithm of García and Tejel [7] or that by the authors of this paper in [18] provides an algorithm for the case where $G$ is 3 -connected since in this case we only need a redundantly rigid augmentation of $G$. In this section we sketch how one can provide an $O\left(|V|^{2}\right)$ time algorithm for Theorem 4.

Theorem 5. Let $G=(V, E)$ be a tight graph. There exists an $O\left(|V|^{2}\right)$ time algorithm that finds a graph $H=(V, F)$ with a minimum cardinality edge set $F$ for which $G+H$ is a globally rigid graph.

Proof (sketch). Note that the tightness of $G$ implies that $|E|=2|V|-3$. Hence the 3-connectivity of $G$ and all cut-pairs and 3-ends of $G$ can be found in $O(|V|)$ time by the algorithm of Hopcroft and Tarjan [9].

The algorithm of Berg and Jordán [4] checks the tightness of $G$ in $O\left(|V|^{2}\right)$ time, moreover, after this it can be used to calculate $T(i j)$ for each pair of vertices $i, j \in V$ in linear time. This fact was used to show that the algorithms in [7] and [18] both provide an optimal redundantly rigid augmentation of $G$ in $O\left(|V|^{2}\right)$ time which completes the proof when the input is 3-connected.

To start the algorithm of Lemma 12, we first need a transversal of $\mathcal{A}^{*}$. (And this is also needed to solve the case where $\left|\mathcal{A}^{*}\right| \leq 3$.) This can be calculated in $O\left(|V|^{2}\right)$ time by using [18, Algorithm 6.1] and [18, Algorithm 6.9] with some slight modifications. We leave the details to the full version of this paper [17].

Since $F$ is a matching throughout the algorithm of Lemma 12 , we need to run the algorithm recursively $O(|V|)$ times, and $G+F$ has $O(|V|)$ edges in each recursive call of the algorithm. To execute the steps of the algorithm, we need to know every cut-pair $(u, v)$ of the graph $G+F$ along with the value of $b_{(u, v)}(G+F)$, and we need to check whether the condition of STEP 5 holds. These all can be checked in $O(|V|)$ time based on the structure provided by the algorithm of Hopcropft and Tarjan [9], see again the full version [17] for more 
details. Finally, Step 7 of the algorithm of Lemma 12 can also be executed in $O(|V|)$ time since we only need to calculate the subgraphs $T\left(x x_{1}\right), T\left(x x_{2}\right)$, $T\left(x x_{3}\right)$, and $T\left(x_{1} x_{3}\right)$ (which needs $O(|V|)$ running time by [4]) for an arbitrarily chosen $x \in N-\left\{x_{1}, x_{2}, x_{3}\right\}$ and check whether $T\left(x x_{1}\right) \cup T\left(x x_{2}\right) \cup T\left(x x_{3}\right)=$ $T\left(x x_{2}\right) \cup T\left(x_{1} x_{3}\right)$.

\section{Concluding remarks}

In this paper, we solved Problem 1 in the case where the input is a tight graph. For general inputs, a constant factor approximation can be given, as follows.

Let us recall the global rigidity pinning problem. In this problem, the goal is to anchor a minimum set of points of a framework such that the resulting framework is globally rigid. In the generic case, pinning can be modelled by adding a complete graph on the anchored vertices to the graph (see [6]). Moreover, instead of a complete graph we can add any globally rigid graph on the anchored vertex set, for example the square graph of a cycle. (A square of a graph arises by connecting all pairs of vertices which has distance at most 2 in the original graph). Notice that the square graph of the cycle on the vertex set $V$ consists of $2|V|$ edges. This way one can see that a constant approximation to the global rigidity pinning problem gives a constant approximation to the global rigidity augmentation problem and vice versa. Fekete and Jordán [6] investigated the global rigidity pinning problem and gave a constant approximation algorithm to it. This implies that there exists a polynomial time constant approximation algorithm to Problem 1 (and it has an approximation ratio at most 4 times more than that of the pinning problem).

For tight input graphs, we can solve the global rigidity pinning problem optimally as follows. It can be shown easily that we must pin at least one vertex from each atom. On the other hand, a complete graph on a transversal of $\mathcal{A}^{*}$ indeed augments $G$ to a globally rigid graph as it contains also the optimal edge set given by Theorem 4 . Thus one vertex from each atom pins the graph optimally. (When $G$ is 3-connected, we may apply the method of [18, Section 8] directly.)

\section{Acknowledgements}

Project no. NKFI-128673 has been implemented with the support provided from the National Research, Development and Innovation Fund of Hungary, financed under the FK_18 funding scheme. The first author was supported by the János Bolyai Research Scholarship of the Hungarian Academy of Sciences and by the UNKP-19-4 and UNKP-20-5 New National Excellence Program of the Ministry for Innovation and Technology. The second author was supported by the European Union, co-financed by the European Social Fund (EFOP-3.6.3-VEKOP-162017-00002). The authors are grateful to Tibor Jordán for his help, the inspiring discussions and his comments. 
Globally rigid augmentation of minimally rigid graphs in $\mathbb{R}^{2}$

\section{References}

1. T.G. Abbot. Generalizations of Kempe's universality theorem. Master's thesis, MIT, 2008. http://web.mit.edu/tabbott/www/papers/mthesis.pdf.

2. B.D.O. Anderson, I. Shames, G. Mao, and B. Fidan. Formal theory of noisy sensor network localization. SIAM J. Discrete Math., 24:684-698, 012010.

3. J. Aspnes, T. Eren, D.K. Goldenberg, A.S. Morse, W. Whiteley, Y.R. Yang, B.D.O. Anderson, and P.N. Belhumeur. A theory of network localization. IEEE Transactions on Mobile Computing, 5(12):1663-1678, Dec 2006.

4. A.R. Berg and T. Jordán. Algorithms for graph rigidity and scene analysis. In Algorithms - ESA 2003, 11th Annual European Symposium, Budapest, Hungary, September 16-19, 2003, Proceedings, pages 78-89, 2003.

5. R. Connelly. Generic global rigidity. Discrete \& Computational Geometry, 33(4):549-63, 2005.

6. Zs. Fekete and T. Jordán. Uniquely localizable networks with few anchors. In S.E. Nikoletseas and J.D.P. Rolim, editors, Algorithmic Aspects of Wireless Sensor Networks, pages 176-183, Berlin, Heidelberg, 2006. Springer Berlin Heidelberg.

7. A. García and J. Tejel. Augmenting the rigidity of a graph in $\mathbb{R}^{2}$. Algorithmica, 59(2):145-168, 2011.

8. S.J. Gortler, A.D. Healy, and D.P. Thurston. Characterizing generic global rigidity. American Journal of Mathematics, 132(4):897-939, 2010.

9. J. Hopcroft and R. Tarjan. Dividing a graph into triconnected components. SIAM J. Comput., 2:135-158, 1973.

10. T.S. Hsu and V. Ramachandran. A linear time algorithm for triconnectivity augmentation. Annual Symposium on Foundations of Computer Science (Proceedings), pages 548-559, 1991.

11. B. Jackson and T. Jordán. Connected rigidity matroids and unique realizations of graphs. J. Comb. Theory, Ser. B, 94:1-29, 2005.

12. B. Jackson and T. Jordán. Graph theoretic techniques in the analysis of uniquely localizable sensor networks. In G. Mao and B. Fidan, editors, Localization algorithms and strategies for wireless sensor networks, pages 146-73. IGI Global, 2009.

13. T. Jordán. On the optimal vertex-connectivity augmentation. J. Comb. Theory, Ser. B, 63:8-20, 1995.

14. T. Jordán. Combinatorial rigidity: Graphs and matroids in the theory of rigid frameworks. In Discrete Geometric Analysis, volume 34 of MSJ Memoirs, pages 33-112. Mathematical Society of Japan, Japan, 2016.

15. T. Jordán and A. Mihálykó. Minimum cost globally rigid subgraphs. In I. Bárány, G.O.H. Katona, and A. Sali, editors, Building Bridges II, volume 28 of Bolyai Society Mathematical Studies, pages 257-278. Springer Berlin Heidelberg, 2019.

16. P. Kaewprapha, J. Li, and N. Puttarak. Network localization on unit disk graphs. In 2011 IEEE Global Telecommunications Conference - GLOBECOM 2011, pages $1-5,2011$.

17. Cs. Király and A. Mihálykó. Globally rigid augmentation of minimally rigid graphs in $\mathbb{R}^{2}$. Technical Report TR-2020-07, Egerváry Research Group, Budapest, 2020. www.cs.elte.hu/egres.

18. Cs. Király and A. Mihálykó. Sparse graphs and an augmentation problem. Technical Report TR-2020-06, Egerváry Research Group, Budapest, 2020. www.cs.elte.hu/egres An extended abstract appeared in Bienstock D., Zambelli G., editors, Integer Programming and Combinatorial Optimization, IPCO 2020, volume 12125 of Lecture Notes in Computer Science, pages 238-251, Springer, Cham, 2020. 
19. G. Laman. On graphs and rigidity of plane skeletal structures. J. Engineering Mathematics, 4:331-340, 1970.

20. H. Pollaczek-Geiringer. Über die Gliederung ebener Fachwerke. ZAMM - Journal of Applied Mathematics and Mechanics, 7(1):58-72, 1927.

21. J.B. Saxe. Embeddability of weighted graphs in $k$-space is strongly NP-hard. Technical report, Computer Science Department, Carnegie-Mellon University, Pittsburgh, PA, 1979.

22. A. So and Y. Ye. Theory of semidefinite programming for sensor network localization. Mathematical Programming, 109:405-414, 012005.

23. W. Whiteley. Some matroids from discrete applied geometry. In J.E. Bonin, J.G. Oxley, and B. Servatius, editors, Matroid Theory, volume 197 of Contemporary Mathematics, pages 171-311. AMS, 1996. 